\title{
Influence of the acoustic energy distribution on the in-liquid sensitivity of thin film electroacoustic
} resonators

\author{
Teona Mirea, Jimena Olivares, Marta Clement, Enrique Iborra
}

\begin{abstract}
In contrast to quartz crystal microbalances, the sensitivity of thin film bulk acoustic wave resonators (FBARs) is strongly dependent on all layers composing the composite structure. Previous studies of the pure mass sensitivity of suspended FBARs, proved that placing low acoustic impedance materials at the sensing surface of the device can enhance their sensitivity by carefully controlling the energy trapping effects. Here we extend those studies by investigating if the in-liquid sensitivity of shear-mode AIN-based solidly mounted resonators (SMRs), working at $2 \mathrm{GHz}$, display a similar dependence on the device configuration (top electrode thickness and material). We use the finite element method (FEM) and experimental results to demonstrate that if one is restricted by the readout circuit to a certain resonant frequency, the sensitivity of the devices (particularly in-liquid sensors or biosensors) can be boosted by proper design while preserving the initial frequency. This is possible since the variations in sensitivity are strongly dependent on the energy distribution within the whole resonant structure.
\end{abstract}

Keywords-AIN-based SMR; finite element method; in-liquid sensing.

\section{INTRODUCTION}

The sensitivity to mass attachment or liquid properties (density and viscosity) of single material electroacoustic resonators, like QCMs, can be derived from Sauerbrey's [1] or Kanazawa and Gordon's [2] equations, being in both cases directly dependent on the resonant frequency and influenced only by the quartz plate properties. Thin film resonators working in the $\mathrm{GHz}$ range can provide with a tremendous increase in sensitivity compared to their QCM antecessors [3]. However, in these type of resonators we cannot consider these simple equations anymore. In this case, to derive the sensitivity, we need to consider multilayered structures where the properties of all layers have an influence [4]. Particularly, the distribution of the acoustic energy within the structure strongly affects the sensitivity at the active surface. In thin film bulk acoustic wave resonators (FBARs) operating in air it has been proved that the addition of a low impedance material (e.g. $\mathrm{SiO}_{2}$ ) on the sensing surface can increase their mass sensitivity [4], which can also be achieved by using low impedance electrodes of appropriate thicknesses [5]. In Ref. [4] it was studied in deep the mass sensitivity of suspended FBARs for both the fundamental and first harmonic modes, and envisaged that the addition of a low acoustic impedance material not only enhances the pure mass sensitivity by the energy trapping effect, but also affects the behavior of the devices in liquid by increasing their dissipation due to a larger portion of energy coupled to the liquid. However, their sensitivity towards liquid properties has not been studied.

Although FBARs working in the $\mathrm{GHz}$ range have overcome the sensitivity of QCMs, in practice the readout circuits can impose frequency restrictions. If such is the case and one cannot go further at higher frequencies, a proper design can increase the sensitivity while preserving the resonant frequency. Our aim here is to prove that the in-liquid sensitivity of solidly mounted resonators (SMRs) can be strongly affected by their design (i.e. electrodes materials and thicknesses) while working at roughly the same resonant frequencies. We assess the in-liquid sensitivity of shear mode SMRs with different structures, particularly towards density and viscosity, by employing the finite element method (FEM) and comparing the results with experimental ones. The study is extended to their mass sensitivity in liquid as an analogy to their biosensing application.

\section{A. Devices}

Our SMRs are based on fully-insulating acoustic reflectors made of seven alternating layers of $\mathrm{SiO}_{2} / \mathrm{AlN}$, on which we deposit a tilted piezoelectric AlN film sandwiched between two electrodes (Fig. 1a). The acoustic reflector was designed to properly confine (reflect) the acoustic energy of the shear mode operating at around $2 \mathrm{GHz}$. Since our purpose was to study the influence of the electrodes and AIN on the device in-liquid sensitivity, the bottom electrode/AlN/top electrode structure was varied keeping always similar frequencies.

To allow testing the response of the devices through a microfluidic system, the electrical contacts to the top electrode of the SMRs were extended to $1.7 \mathrm{~mm}$ far away from the resonator active area to ease contacting the devices outside the fluidic system with the RF probes (Fig. 1b). The bottom electrode located below the extensions of the top electrode was etched to avoid the presence of parasitic capacitances and inductances. For the same purpose, the fully-insulating reflector together with the high resistivity silicon wafer substrate are also pivotal; indeed, reflectors containing metallic or semi-metallic layers lead to the appearance of parasites due 
(a)

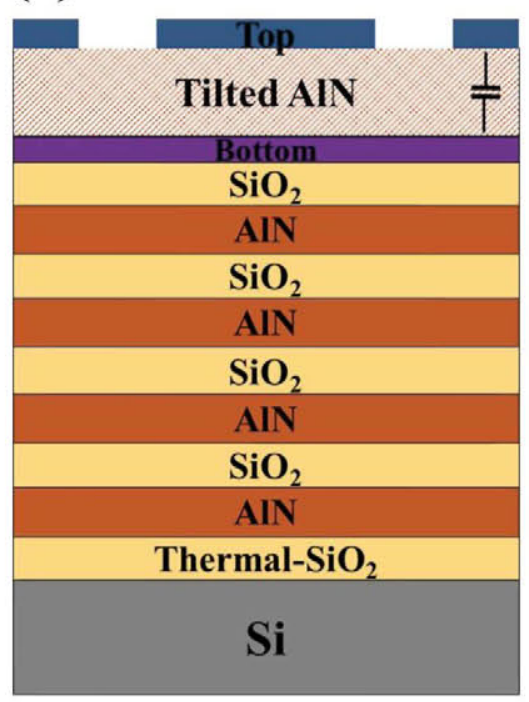

(b)

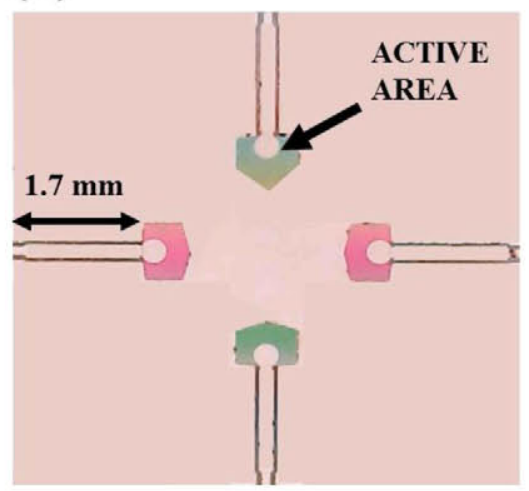

Fig. 1. AlN-based SMRs: (a) cross section sketch, and (b) optical top view of a chip containing 4 devices with extended top electrodes for the microfluidic testing.

to the capacitive coupling of the top electrode with the last layers of the reflector [6].

\section{B. FEM Simulation}

We used a 2D finite element analysis method to assess the in-liquid behavior of our devices. In this case, their response to varying density and viscosity of the contacting liquid as well as their mass sensitivity in liquid. The FEM model was a previously-designed one. A detailed description of this model and its validation can be found elsewhere [7]. It was designed to correctly account for the acoustic coupling to the liquid through shear stresses. The structures we employed for the simulations were single cells with applied lateral periodic conditions (Fig. 2), i.e. we applied periodic continuity on the lateral interfaces since we are not interested here on considering possible spurious modes in the structures but the physical interaction of the shear mode with the liquid interface. The cells include perfectly matched layers (PML) to simulate the absorption of outgoing waves in the liquid domain (liquid

\section{Liquid PML}

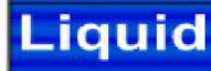

Electrodes

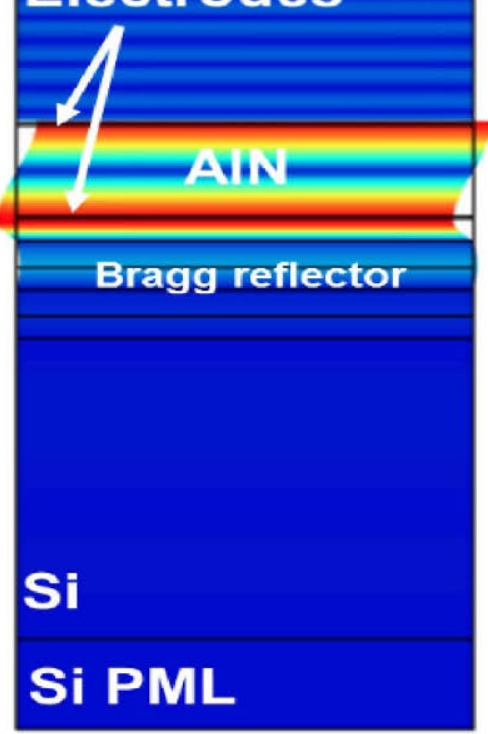

Fig. 2. FEM simulation: SMR single cell with lateral periodic conditions in contact with liquid on top. Liquid and Si PMLs are used to simulate to the complete absortion of the acoustic waves.

PML) and the Si substrate (Si PML) since, for efficiency, we did not consider an entire Si wafer.

\section{Measurements Set-up}

The devices were electrically evaluated with a network analyzer while integrated in a microfluidic system composed of a polydimethylsiloxane (PDMS) chamber where the liquids were recirculated with a peristaltic pump (Fig. 3). As mentioned, the SMR was electrically contacted through its 1.7 mm extensions through a $150 \mu \mathrm{m}$-pitch RF-probe connected to the network analyzer. The device response (variations of its resonant frequency) was monitored by tracking the maximum of the real part of the admittance $(\operatorname{Re}(Y))$ curve through a

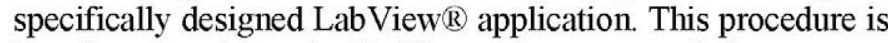
based on acquiring the $\operatorname{Re}(\mathrm{Y})$ maximum around the resonant frequency with a bandwidth of $20 \mathrm{MHz}(\sim 1.5 \mathrm{kHz}$ frequency spacing using 12000 data points) and fitting it to a rational function, which allows tracking the resonant frequency with a noise floor below $1 \mathrm{kHz}$. A typical response curve is shown in Fig. 4, where negative shifts in frequency were induced on the device upon introduction of solutions with different ethylene glycol concentrations in water. As observed, the solutions were sequentially fed starting with the less viscous and dense one. At the end, water was thoroughly flown to clean the device, which was confirmed by the recovery of its initial resonant frequency. 


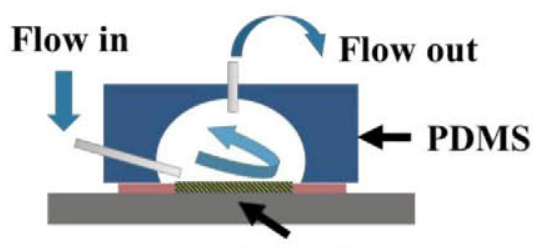

Active Area
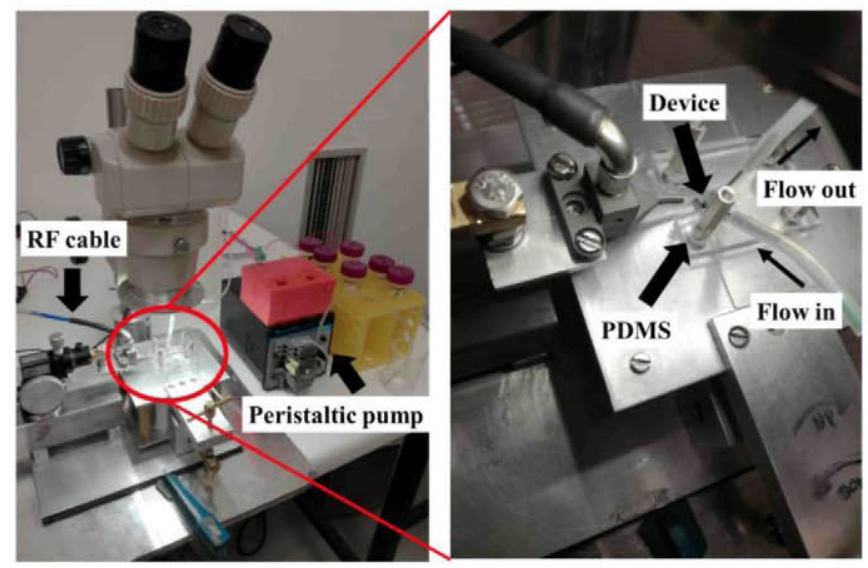

Fig. 3. Measurement set-up with a sketch on top an actual images below.

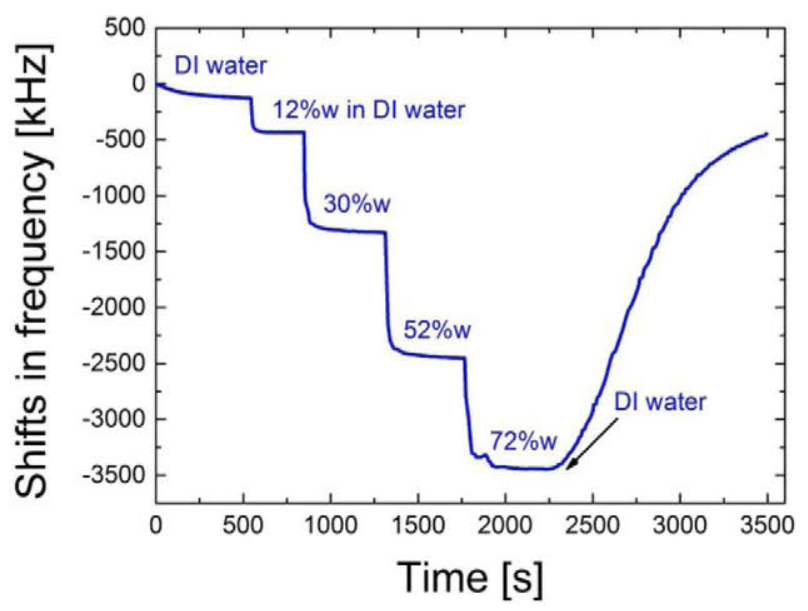

Fig. 4. Typical response of a device with increasing concentration of ethylene glycol $\%$ in pure water $(\%$ w stands for weigth percent of solute in water).

\section{RESULTS AND DISCUSSION}

Three different structures were simulated and experimentally tested. As mentioned, all of them contained the same acoustic reflector but differed on their bottom electrode/AlN/top electrode structure. The structures consisted of:
(a) $100 \mathrm{~nm} \mathrm{Mo} / 900 \mathrm{~nm}$ AlN / $150 \mathrm{~nm} \mathrm{Mo}$
(b) $150 \mathrm{~nm} \mathrm{Mo} / 900 \mathrm{~nm}$ AlN / $100 \mathrm{~nm} \mathrm{Mo}$
(c) $100 \mathrm{~nm} \mathrm{Mo} / 1000 \mathrm{~nm} \mathrm{AlN} \mathrm{/} 100 \mathrm{~nm} \mathrm{Al}$

providing resonant frequencies of $2.007 \mathrm{GHz}, 2.010 \mathrm{GHz}$ and $2.261 \mathrm{GHz}$ for $\mathrm{a}, \mathrm{b}$ and c, respectively.
Fig. 5 shows the simulated and experimental results of all devices towards the different density and viscosity products of the ethylene glycol concentrations from Fig. 4. On one hand, we note a relatively good agreement between simulations and experiments. We attribute the small deviations to errors in the resonant peak tracking due to the presence of spurious modes in some cases. On the other hand, we observe that the sensitivity to density and viscosity is enhanced if (1) we interchange the electrodes thicknesses for cases (a) and (b), and (2) if we employ a lower acoustic impedance top electrode as Al. As for the first case, the reason of the sensitivity improvement at the top electrode surface is a redistribution of the energy within the acoustic cavity, which leads to an increased displacement field, or energy concentration, at the top $100 \mathrm{~nm}$-thick electrode. Regarding the second case, the substitution of the Mo electrode with a lower impedance one (Al), redistributes again the energy within the cavity trapping a greater amount of energy at the Al top surface. This is in agreement with previously reported results where only pure mass sensitivity in air was considered [3]-[5]. Indeed, if one considers the transmission coefficient at the AlN//top electrode interface, which is defined as:

$$
\mathrm{T}=\left(2 \times Z_{\mathrm{AlN}}\right) /\left(Z_{\mathrm{AIN}}+Z_{\mathrm{top}}\right)
$$

being $Z_{\text {AIN }}$ the specific acoustic impedance of $A I N$, and $Z_{\text {top }}$ the one of the top electrode material, it is clear from Table I (acoustic impedance values) that the amplitude of the transmitted wave from AlN to the top electrode is increased when using $\mathrm{Al}(\mathrm{T}=1.42)$, while decreases when using $\mathrm{Mo}(\mathrm{T}=$ 0.72 ). These values explain why we have a greater amount of energy involved in the sensing mechanism when using $\mathrm{Al}$ as top electrode.

Although we can achieve and enhanced sensitivity by properly designing the device, we need to take into account that this increased sensitivity carries also increased losses (lower quality factors) by coupling a greater amount of the energy to the liquid.

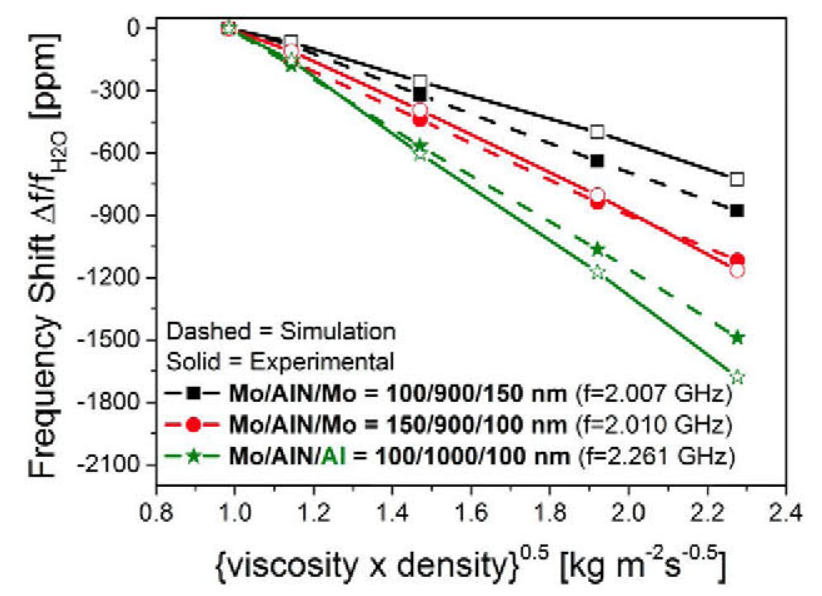

Fig. 5. Simulated and experimental normalized shifts in frequency of the three different structures towards increasing density and viscosity products. 
TABLE I. SHEAR ACOUSTIC IMPEDANCES

\begin{tabular}{|c|c|c|c|}
\hline Material & $\begin{array}{c}\text { Shear acoustic velocity } \\
{[\mathbf{m} / \mathbf{s}]}\end{array}$ & $\begin{array}{c}\text { Density } \\
{\left[\mathbf{k g} / \mathbf{m}^{\mathbf{3}}\right]}\end{array}$ & $Z$ [Rayl] \\
\hline AIN & 6170 & 10300 & $2.03 \cdot 10^{7}$ \\
\hline $\mathrm{Al}$ & 3040 & 3300 & $8.21 \cdot 10^{6}$ \\
\hline $\mathrm{Mo}$ & 3450 & 2700 & $3.55 \cdot 10^{7}$ \\
\hline
\end{tabular}

This is proved in Fig. 6 where we plot experimental values of the inverse of the normalized $\operatorname{Re}(\mathrm{Y})$ peak against density and viscosity for the extreme cases (a) and (c).

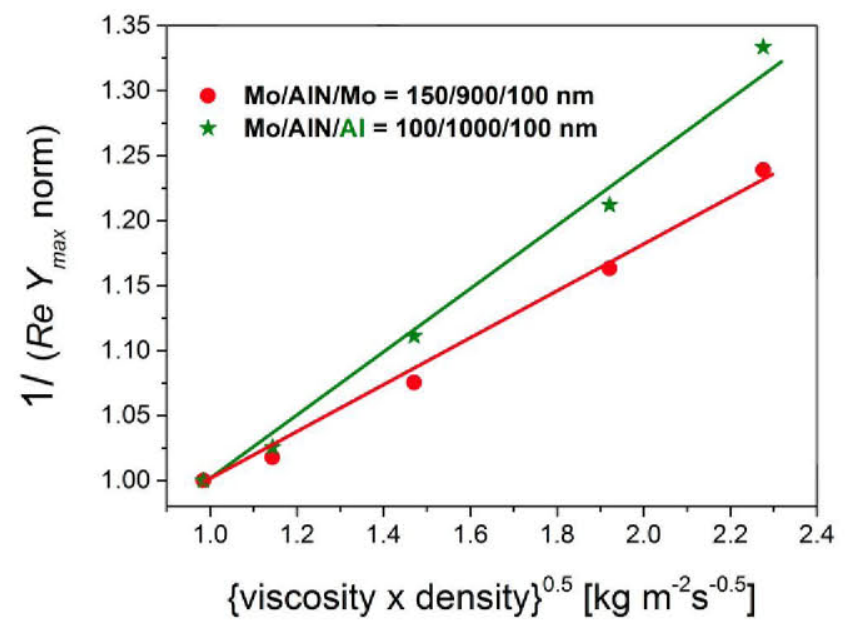

Fig. 6. Experimental measurements of the losses (inverse of the admittance peak normalized to its value in pure water) for structures (a) and (c).

To test the in-liquid mass sensitivity of the devices, experiments with BSA in buffer solutions are ongoing. Nevertheless, we used our model to predict the mass sensitivity results by computing the shift in resonant frequency for each structure towards the deposition of $50 \mathrm{~nm}$ of $\mathrm{SiO}_{2}$ while operating in pure water. We obtained shifts in resonant frequency of $-21.52 \mathrm{MHz},-27.05 \mathrm{MHz}$ and $-49.31 \mathrm{MHz}$, for cases (a), (b) and (c), respectively. Being, as expected, structure (c) more sensitive.

\section{CONCLUSIONS}

We have proved that, although working at the same frequencies, the in-liquid sensitivity of AlN-based shear mode resonators can be strongly affected by their structure. We showed that the sensitivity towards density and viscosity can be increased by reducing the thickness of the top electrode, in this case Mo, or by substituting it with a lower acoustic impedance one, for example Al. This phenomenon is explained by the energy distribution within the whole resonant structure, which gets to be more concentrated at the top sensing surface if the Mo top electrode is reduced or substituted by Al. Our results, obtained by FEM simulations and experimentally verified, are in good agreement with previous works that studied the pure mass sensitivity of suspended FBARs in air.

\section{ACKNOWLEDGMENT}

This work was partially supported by Ministerio de Economía y Competitividad del Gobierno de España through project MAT2013-45957-R.

\section{REFERENCES}

[1] G. Sauerbrey, "Verwendung von Schwingquarzen zur Wagungdiinner Schichten und zur Mikrowagung," Zeitschrift fur Phys., vol. 155, pp. 206-222, 1959.

[2] K. K. Kanazawa and J. G. Gordon, "Frequency of a quartz microbalance in contact with liquid," Anal. Chem., vol. 57, no. 8, pp. 1770-1771, Jul. 1985.

[3] S. Rey-Mermet, R. Lanz, and P. Muralt, "Bulk acoustic wave resonator operating at $8 \mathrm{GHz}$ for gravimetric sensing of organic films," Sensors Actuators B Chem., vol. 114, no. 2, pp. 681-686, Apr. 2006.

[4] G. Wingqvist, V. Yantchev, and I. Katardjiev, "Mass sensitivity of multilayer thin film resonant BAW sensors," Sensors Actuators A Phys., vol. 148, no. 1, pp. 88-95, 2008.

[5] H. Liu, S. Ma, H. Wang, Q. Wang, and L. Qin, "Electrode effects on mass sensitivity of GaN thin film bulk acoustic wave resonator sensors," in 2015 Symposium on Piezoelectricity, Acoustic Waves, and Device Applications (SPAWDA), 2015, pp. 179-183.

[6] M. DeMiguel-Ramos, T. Mirea, J. Olivares, M. Clement, J. Sangrador, E. Iborra, and M. Barba, "Influence of the electrical extensions in AlNBAW resonators for in-liquid biosensors," in 2014 European Frequency and Time Forum (EFTF), 2014, pp. 301-304.

[7] T. Mirea, V. Yantchev, J. Olivares, and E. Iborra, "Influence of liquid properties on the performance of S0-mode Lamb wave sensors II: Experimental validation," Sensors Actuators B Chem., vol. 229, pp. 331337, Jun. 2016. 